REPRESENTATION THEORY

An Electronic Journal of the American Mathematical Society

Volume 15, Pages 370-384 (April 29, 2011)

S 1088-4165(2011)00381-2

\title{
A WEYL MODULE FILTRATION FOR THE VERTEX ALGEBRA OF DIFFERENTIAL OPERATORS
}

\author{
MINXIAN ZHU
}

\begin{abstract}
The aim of this paper is to give a proof of a conjecture stated in a previous paper by the author. We prove the existence of certain filtrations, in the case of rational central charges, for the vertex algebras of differential operators on a Lie group.
\end{abstract}

\section{INTRODUCTION}

Let $\mathfrak{g}$ be a simple complex Lie algebra and $G$ the simply-connected complex Lie group associated to $\mathfrak{g}$. The classical algebra $\mathcal{D}$ of differential operators is generated by the functions and vector fields over $G$. One way to extend this to a vertex algebra is to use the vertex algebroid $\mathcal{A}_{\mathfrak{g}, k}$, as defined in GMS1, GMS2, associated to $\mathfrak{g}$ and a complex parameter $k$. The data for $\mathcal{A}_{\mathfrak{g}, k}$ include functions, vector fields, and 1-forms on $G$, and various structures relating these objects. From $\mathcal{A}_{\mathfrak{g}, k}$, one can construct an associated vertex algebra $U \mathcal{A}_{\mathfrak{g}, k}$, called the enveloping algebra of $\mathcal{A}_{\mathfrak{g}, k}$. We will refer to $U \mathcal{A}_{\mathfrak{g}, k}$ as the vertex algebra of differential operators on $G$ and denote it by $\mathbb{V}_{\mathcal{D}, G}$ or simply $\mathbb{V}$. Let $\hat{\mathfrak{g}}$ be the affine Lie algebra and $h^{\vee}$ the dual Coxeter number of $\mathfrak{g}$. It is shown in [AG] and GMS2 that not only is $\mathbb{V}$ a $\hat{\mathfrak{g}}-$ representation of level $k$, it is also a $\hat{\mathfrak{g}}$-representation of the dual level $\bar{k}=-2 h^{\vee}-k$. Moreover, these two $\hat{\mathfrak{g}}$-actions commute with each other.

When $k$ is irrational, the vertex operator algebra $\mathbb{V}$ decomposes into

$$
\mathbb{V}=\bigoplus_{\lambda \in P^{+}} V_{\lambda, k} \otimes V_{\lambda^{*}, \bar{k}}
$$

as a $\hat{\mathfrak{g}}_{k} \oplus \hat{\mathfrak{g}}_{\bar{k}}$-module ([FS, [Z1]). Here, $P^{+}$is the set of dominant integral weights of $\mathfrak{g}, V_{\lambda, k}$ is the Weyl module induced from $V_{\lambda}$ (the irreducible representation of $\mathfrak{g}$ with highest weight $\lambda$ ) in level $k$, and $V_{\lambda^{*}, \bar{k}}$ is induced from $V_{\lambda}^{*}$ in level $\bar{k}$. In fact, the vertex operators can be constructed using intertwining operators and KnizhnikZamolodchikov equations ([Z1]).

In the case $k \in \mathbb{Q}$, the $\hat{\mathfrak{g}}_{k} \oplus \hat{\mathfrak{g}}_{\bar{k}}$-module structure of $\mathbb{V}$ is more complicated. In the present paper, we prove the existence of filtrations of $\mathbb{V}$ conjectured at the end of [Z1]. More precisely, we will prove

Theorem 1. Let $k \in \mathbb{Q}, k>-h^{\vee}$. The vertex operator algebra $\mathbb{V}$ admits an increasing (resp. a decreasing) filtration of $\hat{\mathfrak{g}}_{k} \oplus \hat{\mathfrak{g}}_{\bar{k}}$-submodules with factors isomorphic to

$$
V_{\lambda, k} \otimes V_{\lambda, \bar{k}}^{c} \quad\left(\text { resp. } V_{\lambda, k}^{c} \otimes V_{\lambda, \bar{k}}\right), \quad \lambda \in P^{+},
$$

where $V_{\lambda, \bar{k}}^{c}$ is the contragradient module of $V_{\lambda, \bar{k}}$.

Received by the editors October 14, 2009 and, in revised form, March 8, 2010.

2010 Mathematics Subject Classification. Primary 17B67, 17B69. 
We need two ingredients to prove the theorem: one is the standard semi-regular module of the affine Lie algebra; the other is the regular representation of the corresponding quantum group at a root of unity.

The semi-regular module was first introduced by A. Voronov in [V] to treat the semi-infinite cohomology of infinite dimensional Lie algebras as a two-sided derived functor of a functor that is neither left nor right exact. It was also studied by S. M. Arkhipov, who defined the associative algebra semi-infinite cohomology in the derived category setting ( $\mathrm{A} 1]$ ), and discovered a deep semi-infinite duality which generalizes the classical bar duality of graded associative algebras ( $\mathrm{A} 2]$ ).

The semi-regular module $S_{\gamma}$ associated to a semi-infinite structure $\gamma$ of $\hat{\mathfrak{g}}$ plays the role of the enveloping algebra of $\hat{\mathfrak{g}}$ in the semi-infinite theory. In particular, $S_{\gamma}$ is a $U(\hat{\mathfrak{g}})$-bimodule. The tensor product of $S_{\gamma}$ and a representation of $\hat{\mathfrak{g}}$ over $U(\hat{\mathfrak{g}})$ is again a $\hat{\mathfrak{g}}$-representation, but the central charge gets shifted by $2 h^{\vee}([\underline{\mathrm{S}}])$. Since the vertex operator algebra $\mathbb{V}=U \mathcal{A}_{\mathfrak{g}, k}$ is a $\hat{\mathfrak{g}}_{k} \oplus \hat{\mathfrak{g}}_{\bar{k}}$-representation (with the subscripts indicating the central charges), the tensor product $S_{\gamma} \otimes_{U(\hat{\mathfrak{g}})} \mathbb{V}$ over $\hat{\mathfrak{g}}_{k}$ becomes a $\hat{\mathfrak{g}}_{-\bar{k}} \oplus \hat{\mathfrak{g}}_{\bar{k}}$-representation. As we will see, $S_{\gamma} \otimes_{U(\hat{\mathfrak{g}})} \mathbb{V}$ can be embedded into $U(\hat{\mathfrak{g}})^{*}$ as a sub-bimodule, and the image consists of matrix coefficients of modules from the Kazhdan-Lusztig category $\mathcal{O}_{\bar{k}+h^{\vee}}$, for $\bar{k}<-h^{\vee}$.

In a series of papers KL1, KL2, KL3, KL4, Kazhdan and Lusztig defined a certain category $\mathcal{O}_{k+h^{\vee}}$ of representations of $\hat{\mathfrak{g}}$ in level $k$, where $k<-h^{\vee}$. They defined a braided tensor structure on it and constructed an equivalence between the tensor category $\mathcal{O}_{k+h^{\vee}}$ and the category of finite dimensional integrable representations of the quantum group with parameter $e^{i \pi /\left(k+h^{\vee}\right)}$ (in the simply-laced case). This motivated the author to study the structure of regular representations of the quantum group at roots of unity ([Z2]).

It is shown in $\mathrm{Z2}$ that the quantum function algebra admits an increasing filtration of sub-bimodules such that the subquotients are isomorphic to the tensor products of the dual of Weyl modules $W_{-\omega_{0} \lambda}^{*} \otimes W_{\lambda}^{*}\left(\omega_{0}\right.$ being the longest element in the Weyl group). Translating this to the affine Lie algebra, means that $S_{\gamma} \otimes_{U(\hat{\mathfrak{g}})} \mathbb{V}$ admits an increasing filtration of $\hat{\mathfrak{g}}_{-\bar{k}} \oplus \hat{\mathfrak{g}}_{\bar{k}}$-submodules with factors isomorphic to the tensor products of the dual and contragredient dual of Weyl modules $V_{-\omega_{0} \lambda, \bar{k}}^{*} \otimes V_{\lambda, \bar{k}}^{c}$.

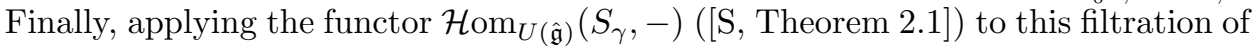
$S_{\gamma} \otimes_{U(\hat{\mathfrak{g}})} \mathbb{V}$, we obtain an increasing filtration of $\hat{\mathfrak{g}}_{k} \oplus \hat{\mathfrak{g}}_{\bar{k}}$-submodules of $\mathbb{V}$, with factors isomorphic to the tensor products of the Weyl module and the contragredient dual of Weyl module $V_{\lambda, k} \otimes V_{\lambda, \bar{k}}^{c}$. The corresponding decreasing filtration is obtained by using the non-degenerate bilinear form on $\mathbb{V}$ constructed in [Z1].

The paper is organized as follows: In Section 2, we recall the definition of the standard semi-regular module $S_{\gamma}$ and the two functors defined with it, following [S]. In Section 3, we embed $S_{\gamma} \otimes_{U(\hat{\mathfrak{g}})} \mathbb{V}$ into the dual of $U(\hat{\mathfrak{g}})$ as a sub-bimodule. In Section 4, we prove the main theorem about the filtrations of the vertex operator algebra $\mathbb{V}$ using results of $[\mathbf{Z 2}$; as a corollary, we compute the semi-infinite cohomology of $\mathbb{V}$ with respect to the diagonal $\hat{\mathfrak{g}}$-action.

Acknowledgments. I am grateful to Dennis Gaitsgory for pointing out Lemma 3.1 to me. I'd also like to thank my advisor I. Frenkel for his constant support and G. Zuckerman for helpful discussions. 


\section{The Standard SEMi-REgular MOdule $S_{\gamma}$ AND AN EQUIVALENCE OF CATEGORIES}

The semi-regular module of a graded Lie algebra with a semi-infinite structure was first introduced by A. Voronov in [V], where it was called the "standard semijective module". It replaces the universal enveloping algebra (and its dual) in the semi-infinite theory and, like the universal enveloping algebra, it possesses left and right (semi-)regular representations. Voronov used semijective complexes and resolutions to define the semi-infinite cohomology of infinite-dimensional Lie algebras as a two-sided derived functor of a functor that is intermediate between the functors of invariants and coinvariants.

In [A2], S. M. Arkhipov generalized the classical bar duality of graded associative algebras to give an alternative construction of the semi-infinite cohomology of associative algebras. Given a graded associative algebra $A$ with a triangular decomposition, he introduced the endomorphism algebra $A^{\sharp}$ of a semi-regular $A$-module $S_{A}$ ([A1]). In the case where $A$ is the universal enveloping algebra of a graded Lie algebra, the algebra $A^{\sharp}$ is also a universal enveloping algebra of a Lie algebra which differs from the previous one by a 1-dimensional central extension (determined by the critical 2-cocycle). In the affine Lie algebra case, he proved that the category of all $\hat{\mathfrak{g}}$-modules with a Weyl filtration in level $k$ is contravariantly equivalent to the analogous category in the dual level $\bar{k}$. This equivalence was obtained directly in $[\mathbf{S}$, where W. Soergel used it to find characters of tilting modules of affine Lie algebras and quantum groups.

Let $\mathfrak{g}$ be a simple complex Lie algebra. Let $\hat{\mathfrak{g}}=\mathfrak{g} \otimes \mathbb{C}\left[t, t^{-1}\right] \oplus \mathbb{C} \underline{c}$ be the affine Lie algebra where the commutator is given by

$$
\left[x_{(m)}, y_{(n)}\right]=[x, y]_{(m+n)}+m \delta_{m+n, 0}(x, y) \underline{c} .
$$

Here $x_{(n)}$ stands for $x \otimes t^{n},($,$) is the normalized Killing form on \mathfrak{g}$, and $\underline{c}$ is the center. Define a $\mathbb{Z}$-grading on $\hat{\mathfrak{g}}$ by

$$
\operatorname{deg} x_{(n)}=n, \quad \operatorname{deg} \underline{c}=0 .
$$

Set

$$
\begin{aligned}
\hat{\mathfrak{g}}_{>0} & =\mathfrak{g} \otimes t \mathbb{C}[t], \quad \hat{\mathfrak{g}}_{<0}=\mathfrak{g} \otimes t^{-1} \mathbb{C}\left[t^{-1}\right], \\
\hat{\mathfrak{g}}_{0} & =\mathfrak{g} \oplus \mathbb{C} \underline{c}, \quad \hat{\mathfrak{g}}_{\geq 0}=\hat{\mathfrak{g}}_{>0} \oplus \hat{\mathfrak{g}}_{0} .
\end{aligned}
$$

We denote the enveloping algebras of $\hat{\mathfrak{g}}, \hat{\mathfrak{g}}_{>0}, \hat{\mathfrak{g}}_{<0}$ by $U, B, N$ respectively. It is obvious that $U, B, N$ inherit $\mathbb{Z}$-gradings from the corresponding Lie algebras.

Definition 2.1 ([S Definition 1.1]). A character $\gamma: \hat{\mathfrak{g}}_{0} \rightarrow \mathbb{C}$ is called a semi-infinite character for $\hat{\mathfrak{g}}$ if we have $\gamma([X, Y])=\operatorname{tr}\left(\operatorname{ad} X\right.$ ad $\left.Y: \hat{\mathfrak{g}}_{0} \rightarrow \hat{\mathfrak{g}}_{0}\right)$ for any $X \in \hat{\mathfrak{g}}_{1}$ and $Y \in \hat{\mathfrak{g}}_{-1}$.

Define a character as follows:

$$
\gamma: \hat{\mathfrak{g}}_{0}=\mathfrak{g} \oplus \mathbb{C} \underline{c} \rightarrow \mathbb{C} ;\left.\quad \gamma\right|_{\mathfrak{g}}=0, \quad \gamma(\underline{c})=2 h^{\vee},
$$

where $h^{\vee}$ is the dual Coxeter number of $\mathfrak{g}$.

Lemma 2.2. The character $\gamma$ defined above is a semi-infinite character for $\hat{\mathfrak{g}}$.

Proof. This is an easy exercise. 
For any two $\mathbb{Z}$-graded vector spaces $M, M^{\prime}$, define $\mathcal{H}_{\mathbb{C}}\left(M, M^{\prime}\right)$ to be the $\mathbb{Z}$ graded vector space with homogeneous components

$$
\mathcal{H o m}_{\mathbb{C}}\left(M, M^{\prime}\right)_{j}=\left\{f \in \operatorname{Hom}_{\mathbb{C}}\left(M, M^{\prime}\right) \mid f\left(M_{i}\right) \subset M_{i+j}^{\prime}\right\} .
$$

Let us recall the definition of the semi-regular module $S_{\gamma}$ from [S, Theorem 1.3]. The graded dual $N^{\circledast}=\bigoplus_{i} N_{i}^{*}$ of $N$ is an $N$-bimodule via the prescriptions

$$
(n f)\left(n_{1}\right)=f\left(n_{1} n\right), \quad(f n)\left(n_{1}\right)=f\left(n n_{1}\right)
$$

for any $n, n_{1} \in N, f \in N^{\circledast}$. One has $N^{\circledast}=\mathcal{H}_{\mathbb{C}}(N, \mathbb{C})$, if $\mathbb{C}$ is equipped with the $\mathbb{Z}$-grading $\mathbb{C}=\mathbb{C}_{0}$. Consider the following sequence of isomorphisms of $\mathbb{Z}$-graded vector spaces:

$$
\mathcal{H} \mathrm{om}_{B}\left(U, \mathbb{C}_{\gamma} \otimes_{\mathbb{C}} B\right) \stackrel{\sim}{\rightarrow} \mathcal{H o m}_{\mathbb{C}}(N, B) \tilde{\leftarrow} N^{\circledast} \otimes_{\mathbb{C}} B \stackrel{\sim}{\rightarrow} N^{\circledast} \otimes_{N} U .
$$

Here $\mathbb{C}_{\gamma}$ is the one-dimensional representation of $\hat{\mathfrak{g}}_{\geq 0}$ defined by the character $\gamma: \hat{\mathfrak{g}}_{0} \rightarrow \mathbb{C}$ and the surjection $\hat{\mathfrak{g}}_{\geq 0} \rightarrow \hat{\mathfrak{g}}_{0} ; \mathbb{C}_{\gamma} \otimes_{\mathbb{C}} B$ is the tensor product of these two (left) $\hat{\mathfrak{g}}_{\geq 0}$-modules. In the leftmost term, $U$ is considered as a $B$-module via left multiplication of $B$ on $U$, and $\mathcal{H} \mathrm{m}_{B}\left(U, \mathbb{C}_{\gamma} \otimes_{\mathbb{C}} B\right)$ is made into a (left) $U$-module via the right multiplication of $U$ onto itself. The first isomorphism is defined as the restriction to $N$ using the identification $\mathbb{C}_{\gamma} \otimes_{\mathbb{C}} B \stackrel{\sim}{\rightarrow} B$ given by $1 \otimes b \mapsto b$. As a vector space, the semi-regular module $S_{\gamma}$ is defined to be

$$
S_{\gamma}=N^{\circledast} \otimes_{\mathbb{C}} B
$$

It is a $U$-bimodule; the left (resp. right) $U$-action on $S_{\gamma}$ is defined via the first two (resp. last) isomorphisms. The semi-infinite character $\gamma$ ensures that these two actions commute.

Lemma 2.3. One has $\underline{c} \cdot s=s \cdot \underline{c}+2 h^{\vee} s$ for any $s \in S_{\gamma}$, where $\underline{c} \cdot s$ and $s \cdot \underline{c}$ stand for the left and right actions of $\underline{c}$ on $s \in S_{\gamma}$.

Proof. This can be easily verified.

Proposition 2.4 ([S, Theorem 1.3]). The map $\iota: N^{\circledast} \hookrightarrow S_{\gamma} ; f \mapsto f \otimes 1$ is an inclusion of $\mathrm{N}$-bimodules. The maps

$$
U \otimes_{N} N^{\circledast} \rightarrow S_{\gamma} ; u \otimes f \mapsto u \cdot \iota(f), \quad N^{\circledast} \otimes_{N} U \rightarrow S_{\gamma} ; f \otimes u \mapsto \iota(f) \cdot u
$$

are bijections.

Remark 2.5. The sequence of isomorphisms

$$
S_{\gamma}=U \otimes_{N} N^{\circledast} \cong B \otimes_{\mathbb{C}} N^{\circledast} \cong \mathcal{H}_{\mathrm{om}_{\mathbb{C}}}(N, B) \stackrel{\sim}{\rightarrow} \mathcal{H}_{\mathrm{om}} \mathrm{m}_{\text {-right }}\left(U, \mathbb{C}_{-\gamma} \otimes B\right)
$$

induces a right $U$-map from $S_{\gamma}$ to $\mathcal{H}_{\text {om }}$-right $\left(U, \mathbb{C}_{-\gamma} \otimes B\right)$.

Let $P^{+}$be the dominant integral weights of $\mathfrak{g}$ and $\lambda \in P^{+}$. Denote by $V_{\lambda, k}=$ $\operatorname{Ind}_{\hat{\mathfrak{g}}>0}^{\hat{\mathfrak{g}}} V_{\lambda}$ the Weyl module induced from the finite-dimensional irreducible representation of $\mathfrak{g}$ with highest weight $\lambda$ in level $k$. Let $V_{\lambda, k}^{*}$ be the graded dual of $V_{\lambda, k}$, on which $\hat{\mathfrak{g}}$ acts by $X f(v)=-f(X v)$ for any $X \in \hat{\mathfrak{g}}, f \in V_{\lambda, k}^{*}$ and $v \in V_{\lambda, k}$.

Let $\mathcal{M}$ (resp. $\mathcal{K}$ ) denote the category of all $\mathbb{Z}$-graded representations of $\hat{\mathfrak{g}}$ that are isomorphic over $N$ to finite direct sums of grading-shifted copies of $N$ (resp. $\left.N^{\circledast}\right)$. In fact, $\mathcal{M}$ (resp. $\mathcal{K}$ ) consists precisely of those $\mathbb{Z}$-graded $\hat{\mathfrak{g}}$-modules which admit a finite Weyl (resp. dual Weyl) flag ([S, Remarks 2.4]). 
Proposition 2.6 ([S, Theorem 2.1]). The functor $S_{\gamma} \otimes_{U}-: \mathcal{M} \rightarrow \mathcal{K}$ defines an equivalence of categories with inverse $\mathcal{H}_{\mathrm{om}}\left(S_{\gamma},-\right)$, such that short exact sequences correspond to short exact sequences.

Proof. Note that

$$
S_{\gamma} \otimes_{U}-\cong N^{\circledast} \otimes_{N}-\quad \text { and } \quad \mathcal{H o m}_{U}\left(S_{\gamma},-\right) \cong \mathcal{H o m}_{N}\left(N^{\circledast},-\right)
$$

by Proposition 2.4

Proposition 2.7. Let $E$ be a $\mathbb{Z}$-graded $B$-module with the $\mathbb{Z}$-grading bounded from below. The functor $S_{\gamma} \otimes_{U}-$ maps $U \otimes_{B} E$ to $\mathcal{H o m}_{B}\left(U, \mathbb{C}_{\gamma} \otimes E\right)$.

Proof. This is similar to the construction of the semi-regular module $S_{\gamma}$. Consider the following sequence of isomorphisms of $\mathbb{Z}$-graded vector spaces:

$$
S_{\gamma} \otimes_{U}\left(U \otimes_{B} E\right) \cong N^{\circledast} \otimes_{\mathbb{C}} E \stackrel{\sim}{\rightarrow} \mathcal{H o m}_{\mathbb{C}}(N, E) \check{\leftarrow} \mathcal{H o m}_{B}\left(U, \mathbb{C}_{\gamma} \otimes E\right) .
$$

It is straightforward to check that, under these isomorphisms, the (left) $U$-module structure of $S_{\gamma} \otimes_{U}\left(U \otimes_{B} E\right)$ agrees with that of $\mathcal{H o m}_{B}\left(U, \mathbb{C}_{\gamma} \otimes E\right)$.

Remark 2.8. In general, for any $\mathbb{Z}$-graded $B$-module $E^{\prime}$, the inclusion

$$
S_{\gamma} \otimes_{U}\left(U \otimes_{B} E^{\prime}\right) \cong N^{\circledast} \otimes_{\mathbb{C}} E^{\prime} \hookrightarrow \mathcal{H} \operatorname{om}_{B}\left(U, \mathbb{C}_{\gamma} \otimes E^{\prime}\right)
$$

is a $U$-map.

Proposition 2.9. Let $F$ be a $\mathbb{Z}$-graded $B$-module with the $\mathbb{Z}$-grading bounded from above. Then the functor $\mathcal{H}_{\text {om }}\left(S_{\gamma},-\right)$ maps $\mathcal{H}_{\text {om }}(U, F)$ to $U \otimes_{B}\left(\mathbb{C}_{-\gamma} \otimes F\right)$.

Proof. The isomorphisms of vector spaces

$$
\begin{aligned}
& \mathcal{H}_{\operatorname{om}_{U}}\left(S_{\gamma}, \mathcal{H} \operatorname{om}_{B}(U, F)\right) \cong \mathcal{H} \operatorname{om}_{N}\left(N^{\circledast}, \mathcal{H}_{\mathbb{C}}(N, F)\right) \\
& \cong \mathcal{H} \operatorname{om}_{\mathbb{C}}\left(N^{\circledast}, F\right) \cong N \otimes_{\mathbb{C}} F \cong U \otimes_{B}\left(\mathbb{C}_{-\gamma} \otimes F\right)
\end{aligned}
$$

induce an isomorphism of vector spaces:

$$
\alpha: U \otimes_{B}\left(\mathbb{C}_{-\gamma} \otimes F\right) \stackrel{\sim}{\rightarrow} \mathcal{H o m}_{U}\left(S_{\gamma}, \mathcal{H} \operatorname{om}_{B}(U, F)\right) .
$$

Moreover, $\alpha$ agrees with the composition of (left) $U$-maps

$U \otimes_{B}\left(\mathbb{C}_{-\gamma} \otimes F\right) \rightarrow \mathcal{H o m}_{U}\left(S_{\gamma}, S_{\gamma} \otimes_{U}\left(U \otimes_{B}\left(\mathbb{C}_{-\gamma} \otimes F\right)\right)\right) \rightarrow \mathcal{H o m}_{U}\left(S_{\gamma}, \mathcal{H o m}_{B}(U, F)\right)$.

Hence $\alpha$ is a $U$-isomorphism.

In particular, $S_{\gamma} \otimes_{U}$ - transforms Weyl modules to the dual of Weyl modules, and $\mathcal{H o m}_{U}\left(S_{\gamma},-\right)$ transforms the latter to the former (both with a level shift).

Corollary 2.10. We have $S_{\gamma} \otimes_{U} V_{\lambda, k} \cong V_{\lambda^{*}, \bar{k}}^{*}$ and $\mathcal{H} o m_{U}\left(S_{\gamma}, V_{\lambda, \bar{k}}^{*}\right) \cong V_{\lambda^{*}, k}$, where $\lambda^{*}$ denotes the highest weight of $V_{\lambda}^{*}$.

Proof. Note that $U \otimes_{B} V_{\lambda}=V_{\lambda, k}$ and $\mathcal{H} \mathrm{om}_{B}\left(U, \mathbb{C}_{\gamma} \otimes V_{\lambda}\right) \cong V_{\lambda^{*}, \bar{k}}^{*}$ if $\underline{c}$ acts on $V_{\lambda}$ as scalar multiplication by $k$. 


\section{Realization of $S_{\gamma} \otimes_{U} \mathbb{V}$ inside $U^{*}$}

Fix a complex number $k$, and let $\mathbb{V}=U \mathcal{A}_{\mathfrak{g}, k}$ be the vertex operator algebra associated to the vertex algebroid $\mathcal{A}_{\mathfrak{g}, k}$ (see [AG], GMS1, GMS2, [Z1]). Note that in [Z1], we used $\mathbb{V}$ to denote the vertex operator algebra for generic values of $k \notin \mathbb{Q}$. Here, we adopt this notation with no restriction on $k$. We will regard $\mathbb{V}$ as nonpositively graded, i.e. taking the opposite of the grading defined by the conformal weights of the vertex operator algebra $\mathbb{V}$.

The vertex operator algebra $\mathbb{V}$ admits two commuting actions of $\hat{\mathfrak{g}}$ in dual levels $k, \bar{k}=-2 h^{\vee}-k$. It follows from Lemma 2.3 that $S_{\gamma} \otimes_{U} \mathbb{V}$, using the $\hat{\mathfrak{g}}_{k}$-module structure of $\mathbb{V}$ in the tensor product, becomes a $\hat{\mathfrak{g}}_{-\bar{k}} \oplus \hat{\mathfrak{g}}_{\bar{k}}$-representation. Define

$$
U(\hat{\mathfrak{g}}, k)=U(\hat{\mathfrak{g}}) /(\underline{c}-k) U(\hat{\mathfrak{g}}) .
$$

Our goal is to construct an embedding of $U$-bimodules

$$
\Phi: S_{\gamma} \otimes_{U} \mathbb{V} \hookrightarrow U(\hat{\mathfrak{g}}, \bar{k})^{*}
$$

More precisely, $\Phi$ satisfies

$$
\Phi\left(u^{1} \cdot m\right)=\Phi(m) \cdot \bar{u}, \quad \Phi\left(u^{2} \cdot m\right)=u \cdot \Phi(m)
$$

for any $u \in U, m \in S_{\gamma} \otimes_{U} \mathbb{V}$. Here $u^{\mathbf{1}} \cdot m, u^{\mathbf{2}} \cdot m$ denote the $\hat{\mathfrak{g}}_{-\bar{k}^{-}}$and $\hat{\mathfrak{g}}_{\bar{k}}$-actions on $S_{\gamma} \otimes_{U} \mathbb{V}$, respectively;

$$
\text { - }: U \rightarrow U ; \quad u \mapsto \bar{u}
$$

is the anti-involution of $U$ determined by $-\mathrm{Id}: \hat{\mathfrak{g}} \rightarrow \hat{\mathfrak{g}}$; the dual space $U^{*}$ is made into a $U$-bimodule via the recipes

$$
(u \cdot g)\left(u_{1}\right)=g\left(u_{1} u\right) \quad \text { and } \quad(g \cdot u)\left(u_{1}\right)=g\left(u u_{1}\right)
$$

for any $u, u_{1} \in U, g \in U^{*}$.

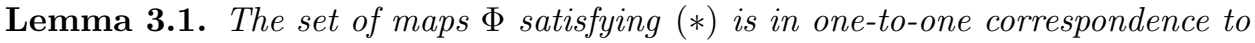
the set of maps $\phi: S_{\gamma} \otimes_{U} \mathbb{V} \rightarrow \mathbb{C}$ such that

$$
\phi\left(\bar{u}^{1} \cdot m\right)=\phi\left(u^{2} \cdot m\right)
$$

for any $u \in U$ and $m \in S_{\gamma} \otimes_{U} \mathbb{V}$.

Proof. Given $\Phi$, we define $\phi$ to be the composition of $\Phi$ and the evaluation at $1 \in U$. Conversely given $\phi$, define $\Phi(m)(u)=\phi\left(u^{2} \cdot m\right)$.

Let $\mathbb{B}=\bigoplus_{i \leq 0} \mathbb{B}_{i}$ (denoted by " $B$ " with opposite grading in [Z1]) be the commutative vertex subalgebra of $\mathbb{V}$ generated by $A$, where $A$ is the commutative algebra of regular functions on the simply-connected complex Lie group $G$ with Lie algebra $\mathfrak{g}$. Recall that $\mathbb{B}$ is closed under the actions of $U\left(\hat{\mathfrak{g}}_{\geq 0}, k\right)$ and $U\left(\hat{\mathfrak{g}}_{\geq 0}, \bar{k}\right)$. As a $\hat{\mathfrak{g}}_{k}$-module, we have

$$
\mathbb{V} \cong U \otimes_{B} \mathbb{B} \cong N \otimes_{\mathbb{C}} \mathbb{B}
$$

(see e.g. [Z1, Proposition 3.14]). Since $S_{\gamma} \cong N^{\circledast} \otimes_{N} U$ as a right $U$-module, we have

$$
S_{\gamma} \otimes_{U} \mathbb{V} \cong N^{\circledast} \otimes_{N} \mathbb{V} \cong N^{\circledast} \otimes_{\mathbb{C}} \mathbb{B} .
$$

Define a functional $\epsilon: \mathbb{B} \rightarrow \mathbb{C}$ as follows: $\left.\epsilon\right|_{\mathbb{B}_{\leq-1}}=0$ and its restriction to $\mathbb{B}_{0}=A$ is the evaluation of functions at the identity.

Define

$$
\phi: S_{\gamma} \otimes_{U} \mathbb{V}\left(\cong N^{\circledast} \otimes_{\mathbb{C}} \mathbb{B}\right) \rightarrow \mathbb{C}
$$


as follows:

$$
\phi(f \otimes b)=f(1) \epsilon(b)
$$

for any $f \in N^{\circledast}, b \in \mathbb{B}$.

We will show that $\phi$ satisfies the condition (**) in Lemma 3.1. Let

$$
\Theta: S_{\gamma} \otimes_{U} \mathbb{V} \rightarrow \mathcal{H} \operatorname{mom}_{B}\left(U, \mathbb{C}_{\gamma} \otimes \mathbb{B}\right)
$$

be the (left) $U$-map described in Remark 2.8 (taking $E^{\prime}=\mathbb{B}$ ). Here, $\mathbb{B}$ is regarded as a left $B$-module via the $U\left(\hat{\mathfrak{g}}_{\geq 0}, k\right)$-action on $\mathbb{B}$, and $\Theta$ is a $U(\hat{\mathfrak{g}},-\bar{k})$-map.

Lemma 3.2. For any $f \otimes b \in S_{\gamma} \otimes_{U} \mathbb{V}$, we have $\phi(f \otimes b)=\epsilon \Theta(f \otimes b)(1)$.

Proof. This is obvious.

Multiplication induces an isomorphism of vector spaces $N \otimes_{\mathbb{C}} B \cong U$, hence any $u \in U$ can be written as $u=u_{<0} u_{\geq 0}$ with $u_{<0} \in N$ and $u_{\geq 0} \in B$.

Let $\beta: B \rightarrow B$ be the automorphism which restricts to $\hat{\mathfrak{g}}_{\geq 0}$ as $X \mapsto \gamma(X)+X$.

Lemma 3.3. For any $u=u_{<0} u_{\geq 0} \in U, f \otimes b \in N^{\circledast} \otimes_{\mathbb{C}} \mathbb{B}$, we have

$$
\phi\left(\bar{u}^{\mathbf{1}} \cdot(f \otimes b)\right)=f\left(\overline{u_{<0}}\right) \epsilon\left(\beta\left(\overline{u_{\geq 0}}\right)^{\mathbf{1}} \cdot b\right)
$$

where $\beta\left(\overline{u_{\geq 0}}\right)^{\mathbf{1}} \cdot b$ denotes the $U\left(\hat{\mathfrak{g}}_{\geq 0}, k\right)$-action on $\mathbb{B}$.

Proof. By Lemma 3.2 and the definition of the $U$-map $\Theta$, we have

$$
\begin{aligned}
\phi\left(\bar{u}^{\mathbf{1}} \cdot(f \otimes b)\right) & =\epsilon \Theta\left(\bar{u}^{\mathbf{1}} \cdot(f \otimes b)\right)(1)=\epsilon(\bar{u} \cdot \Theta(f \otimes b)(1)) \\
& =\epsilon(\Theta(f \otimes b)(\bar{u}))=\epsilon\left(\Theta(f \otimes b)\left(\overline{u_{\geq 0}} \overline{u_{<0}}\right)\right)=\epsilon\left(f\left(\overline{u_{<0}}\right) \beta\left(\overline{u_{\geq 0}}\right)^{\mathbf{1}} \cdot b\right) \\
& =f\left(\overline{u_{<0}}\right) \epsilon\left(\beta\left(\overline{u_{\geq 0}}\right)^{\mathbf{1}} \cdot b\right) .
\end{aligned}
$$

Following GMS2, Z1, let $\tau_{i}$ be an orthonormal basis of $\mathfrak{g}$ with respect to the normalized Killing form (,). Let $C_{i j k}$ be the structure constants determined by $\left[\tau_{i}, \tau_{j}\right]=C_{i j k} \tau_{k}$. We identify $\mathfrak{g}$ with the tangent space of the identity of $G$. Let $\tau_{i}^{L}$ (resp. $\tau_{i}^{R}$ ) be the left (resp. right) invariant vector fields valued $\tau_{i}$ (resp. $-\tau_{i}$ ) at the identity. There exist regular functions $a^{i j} \in A$ such that $\tau_{i}^{R}=a^{i j} \tau_{j}^{L}$ and $\epsilon\left(a^{i j}\right)=-\delta_{i j}$.

Lemma 3.4. For any $u_{<0} \in N$ and $f \otimes b \in N^{\circledast} \otimes_{\mathbb{C}} \mathbb{B}$, we have

$$
\phi\left(u_{<0}^{2} \cdot(f \otimes b)\right)=f\left(\overline{u_{<0}}\right) \epsilon(b) .
$$

Proof. Suppose $u_{<0}=\tau_{i_{1}\left(-m_{1}\right)} \tau_{i_{2}\left(-m_{2}\right)} \cdots \tau_{i_{p}\left(-m_{p}\right)}$ where $m_{1}, \ldots, m_{p}>0$. By [Z1, Lemma 3.12 (9), Lemma 3.33], it is not difficult to see that

$$
u_{<0}^{\mathbf{2}} \cdot b \equiv \sum_{j_{1}, \ldots, j_{p}} \tau_{j_{p}}^{\mathbf{1}} \underset{\left(-m_{p}\right)}{\mathbf{1}} \ldots \tau_{j_{1}\left(-m_{1}\right)}^{\mathbf{1}} a_{(-1)}^{\boldsymbol{i}_{1} j_{1}} \ldots a_{(-1)}^{\boldsymbol{i}_{p} j_{p}} b\left(\bmod U\left(\hat{\mathfrak{g}}_{<0}, k\right) \otimes \mathbb{B}_{\leq-1}\right)
$$

where ${ }^{\mathbf{1}},{ }^{\mathbf{2}}$ denote the $U(\hat{\mathfrak{g}}, k)$ - and $U(\hat{\mathfrak{g}}, \bar{k})$-actions on $\mathbb{B}$, respectively. It follows that

$$
u_{<0}^{2} \cdot(f \otimes b) \equiv \sum_{j_{1}, \ldots, j_{p}} f \cdot \tau_{j_{p}\left(-m_{p}\right)} \cdots \tau_{j_{1}\left(-m_{1}\right)} \otimes a_{(-1)}^{i_{1} j_{1}} \ldots a_{(-1)}^{i_{p} j_{p}} b\left(\bmod N^{\circledast} \otimes \mathbb{B}_{\leq-1}\right) .
$$


Since $\left.\epsilon\right|_{\mathbb{B}_{\leq-1}}=0$ and $\epsilon\left(a^{i j}\right)=-\delta_{i j}$, we have

$$
\begin{aligned}
\phi\left(u_{<0}^{2} \cdot(f \otimes b)\right) & =f\left(\tau_{j_{p}\left(-m_{p}\right)} \cdots \tau_{j_{1}\left(-m_{1}\right)}\right) \epsilon\left(a_{(-1)}^{i_{1} j_{1}} \cdots a_{(-1)}^{i_{p} j_{p}} b\right) \\
& =f\left(\tau_{j_{p}\left(-m_{p}\right)} \cdots \tau_{j_{1}\left(-m_{1}\right)}\right)\left(-\delta_{i_{1} j_{1}}\right) \cdots\left(-\delta_{i_{p} j_{p}}\right) \epsilon(b) \\
& =f\left(\tau_{i_{p}\left(-m_{p}\right)} \cdots \tau_{i_{1}\left(-m_{1}\right)}\right)(-1)^{p} \epsilon(b)=f\left(\overline{u_{<0}}\right) \epsilon(b) .
\end{aligned}
$$

Corollary 3.5. For any $u=u_{<0} u_{\geq 0} \in U$ and $f \otimes b \in N^{\circledast} \otimes_{\mathbb{C}} \mathbb{B}$, we have

$$
\phi\left(u^{2} \cdot(f \otimes b)\right)=f\left(\overline{u_{<0}}\right) \epsilon\left(u_{\geq 0}^{2} \cdot b\right)
$$

where $u_{\geq 0}^{2} \cdot b$ denotes the $U\left(\hat{\mathfrak{g}}_{\geq 0}, \bar{k}\right)$-action on $\mathbb{B}$.

Proof. It follows from the above lemma since $u^{2} \cdot(f \otimes b)=u_{<0}^{2} \cdot\left(f \otimes u_{\geq 0}^{2} \cdot b\right)$ and $u_{\geq 0}^{2} \cdot b \in \mathbb{B}$.

Based on Lemma 3.3 and Corollary 3.5, the condition $(* *)$ for $\phi$ in Lemma 3.1 is reduced to the following.

Lemma 3.6. For any $u_{\geq 0} \in B$ and $b \in \mathbb{B}$, we have

$$
\epsilon\left(\beta\left(u_{\geq 0}\right)^{\mathbf{1}} \cdot b\right)=\epsilon\left({\overline{u_{\geq 0}}}^{2} \cdot b\right)
$$

where $\beta\left(u_{\geq 0}\right)^{\mathbf{1}} \cdot b,{\overline{u_{\geq 0}}}^{2} \cdot b$ denote the $U\left(\hat{\mathfrak{g}}_{\geq 0}, k\right)$ - and $U\left(\hat{\mathfrak{g}}_{\geq 0}, \bar{k}\right)$-actions on $\mathbb{B}$.

Proof. By [Z1, Lemma 3.14 (10)], we have

$$
\tau_{j(n)}^{\mathbf{1}} \cdot b=\sum_{i} \sum_{p \geq 0} a_{(-1-p)}^{i j} \tau_{i(n+p)}^{\mathbf{2}} \cdot b
$$

for any $n \geq 0, b \in \mathbb{B}$. Since $\left.\epsilon\right|_{B_{\leq-1}}=0$, we have

$$
\epsilon\left(\tau_{j(n)}^{\mathbf{1}} \cdot b\right)=\sum_{i} \epsilon\left(a_{(-1)}^{i j} \tau_{i(n)}^{2} \cdot b\right)=\sum_{i}\left(-\delta_{i j}\right) \epsilon\left(\tau_{i(n)}^{2} \cdot b\right)=-\epsilon\left(\tau_{j(n)}^{2} \cdot b\right) .
$$

Since the $U\left(\hat{\mathfrak{g}}_{\geq 0}, k\right)$ - and $U\left(\hat{\mathfrak{g}}_{\geq 0}, \bar{k}\right)$-actions on $\mathbb{B}$ commute, it is not difficult to see that for any $u_{\geq 0}=\tau_{j_{1}\left(n_{1}\right)} \cdots \tau_{j_{q}\left(n_{q}\right)}$ where $n_{i} \geq 0$, we have

$$
\epsilon\left(\beta\left(u_{\geq 0}\right)^{\mathbf{1}} \cdot b\right)=\epsilon\left(u_{\geq 0}^{1} \cdot b\right)=\epsilon\left({\overline{u_{\geq 0}}}^{2} \cdot b\right) .
$$

We also have

$$
\epsilon\left(\beta(\underline{c})^{1} \cdot b\right)=\epsilon\left(\left(\underline{c}+2 h^{\vee}\right)^{1} \cdot b\right)=\epsilon\left(\left(k+2 h^{\vee}\right) b\right)=\epsilon(-\bar{k} b)=\epsilon\left(\underline{c}^{2} \cdot b\right) .
$$

The lemma is proved.

Once we have verifed that $\phi$ satisfies $(* *)$, by Lemma 3.1, we can define a map

$$
\Phi: S_{\gamma} \otimes_{U} \mathbb{V} \rightarrow U^{*}
$$

as follows: For any $f \in N^{\circledast}, b \in \mathbb{B}, u=u_{<0} u_{\geq 0} \in U$,

$$
\Phi(f \otimes b)\left(u_{<0} u_{\geq 0}\right)=f\left(\overline{u_{<0}}\right) \epsilon\left(u_{\geq 0}^{2} \cdot b\right) .
$$

In fact, $\Phi(f \otimes b) \in U(\hat{\mathfrak{g}}, \bar{k})^{*}$.

Theorem 3.7. $\Phi$ satisfies the following:

$$
\begin{aligned}
& \Phi\left(u^{1} \cdot(f \otimes b)\right)=\Phi(f \otimes b) \cdot \bar{u}, \\
& \Phi\left(u^{2} \cdot(f \otimes b)\right)=u \cdot \Phi(f \otimes b) .
\end{aligned}
$$

where ${ }^{\mathbf{1}},{ }^{\mathbf{2}}$ stand for the $\hat{\mathfrak{g}}_{-\bar{k}}$ - and $\hat{\mathfrak{g}}_{\bar{k}}$-actions on $S_{\gamma} \otimes_{U} \mathbb{V}$, respectively. 
Proof. It follows from Lemmas 3.1, 3.3, 3.6 and Corollary 3.5.

Remark 3.8. Following the notations in [Z1], let $\left\{\widetilde{\omega}_{i}\right\}$ be right-invariant 1-forms dual to $\left\{\tau_{i}^{R}\right\}$, and let $\widetilde{\mathbb{B}_{0}}$ be the linear span of elements of the form $\partial^{\left(j_{1}\right)} \widetilde{\omega}_{i_{1}} \cdots \partial^{\left(j_{n}\right)} \widetilde{\omega}_{i_{n}}$, then $\mathbb{B}=A \otimes \widetilde{\mathbb{B}_{0}}$. There is a non-degenerate pairing between $U\left(\hat{\mathfrak{g}}_{>0}\right)$ and $\widetilde{\mathbb{B}_{0}}$, defined by $\left(u_{>0}, \tilde{b}\right)=\epsilon\left(u_{>0}^{2} \cdot \tilde{b}\right)$, via which $\widetilde{\mathbb{B}_{0}}$ can be identified with $U\left(\hat{\mathfrak{g}}_{>0}\right)^{\circledast}$, the graded dual of $U\left(\hat{\mathfrak{g}}_{>0}\right)$. The regular functions $A$ can be identified with the Hopf dual $U(\mathfrak{g})_{\text {Hopf }}^{*}$, which is a subalgebra of $U(\mathfrak{g})^{*}$ defined by

$$
U(\mathfrak{g})_{\text {Hopf }}^{*}=\left\{\phi \in U(\mathfrak{g})^{*} \mid \operatorname{Ker} \phi \text { contains a two-sided ideal } J \subset U(\mathfrak{g})\right.
$$

of finite codimension $\}$.

It is not hard to see that $\epsilon\left(u_{0}^{2} \cdot u_{>0}^{2} \cdot a \tilde{b}\right)=\epsilon\left(u_{0}^{2} \cdot a\right) \epsilon\left(u_{>0}^{2} \cdot \tilde{b}\right)$ for any $u_{0} \in U(\mathfrak{g}), u_{>0} \in$ $U\left(\hat{\mathfrak{g}}_{>0}\right), a \in A$ and $\tilde{b} \in \widetilde{\mathbb{B}_{0}}$. Hence, we have

$S_{\gamma} \otimes_{U} \mathbb{V} \cong N^{\circledast} \otimes \mathbb{B} \cong N^{\circledast} \otimes A \otimes \widetilde{\mathbb{B}_{0}} \cong U\left(\hat{\mathfrak{g}}_{<0}\right)^{\circledast} \otimes U(\mathfrak{g})_{\text {Hopf }}^{*} \otimes U\left(\hat{\mathfrak{g}}_{>0}\right)^{\circledast} \subset U(\hat{\mathfrak{g}}, \bar{k})^{*}$ and $\Phi$ is injective.

\section{Filtrations of the vertex operator Algebra $\mathbb{V}$}

Fix $k \in \mathbb{Q}, k>-h^{\vee}$; set $\varkappa=k+h^{\vee}>0$. We denote by $L_{\lambda, \bar{k}}$ the irreducible quotient of the Weyl module $V_{\lambda, \bar{k}}$.

Definition 4.1 ([KL1, Definition 2.15]). Let $\mathcal{O}_{-\varkappa}$ be the full subcategory of the category of $\hat{\mathfrak{g}}_{\hat{k}}$-modules, which admits a finite composition series with factors of the form $L_{\lambda, \bar{k}}$ for various $\lambda \in P^{+}$.

Let us recall some basic facts about $\mathcal{O}_{-\varkappa}$. The $\mathbb{Z}_{>0}$-grading on $\hat{\mathfrak{g}}_{>0}$ induces an $\mathbb{N}$-grading on its enveloping algebra: $U\left(\hat{\mathfrak{g}}_{>0}\right)=\bigoplus_{n>0} U\left(\hat{\mathfrak{g}}_{>0}\right)_{n}$. For any $V \in \mathcal{O}_{-\varkappa}$, $v \in V$, there exists an $n_{1} \in \mathbb{N}$ such that $U\left(\hat{\mathfrak{g}}_{>0}\right)_{n_{1}} \cdot v=0$. A module $\mathcal{N}$ over $\mathfrak{g} \otimes \mathbb{C}[t]$ is said to be a nil-module if $\operatorname{dim}_{\mathbb{C}} \mathcal{N}<\infty$ and there exists an $n \geq 1$ such that $U\left(\hat{\mathfrak{g}}_{>0}\right)_{n} \mathcal{N}=0$. We can regard $\mathcal{N}$ as a $\hat{\mathfrak{g}}_{\geq 0}$-module by letting $\underline{c}$ act as multiplication by $\bar{k}$. We call the induced module $\mathcal{N}_{\bar{k}}=\operatorname{Ind}_{\hat{\mathfrak{g}} \geq 0}^{\hat{\mathfrak{g}}} \mathcal{N}$ a generalized Weyl module.

Proposition 4.2 ([KL1, Theorem 2.22]). $A \hat{\mathfrak{g}}_{\bar{k}}$-module $V$ is in $\mathcal{O}_{-\varkappa}$ if and only if $V$ is a quotient of a generalized Weyl module.

Given $V \in \mathcal{O}_{-\varkappa}$, let $\bar{L}_{0}: V \rightarrow V$ be the Sugawara operator defined by

$$
\bar{L}_{0} v=-\frac{1}{\varkappa} \sum_{j>0} \sum_{i} \tau_{i(-j)} \tau_{i(j)} v-\frac{1}{2 \varkappa} \sum_{i} \tau_{i(0)} \tau_{i(0)} v
$$

where $\left\{\tau_{i}\right\}$ is an orthonormal basis of $\mathfrak{g}$ with respect to the normalized Killing form. Note that this operator is well defined and locally finite. Let $V_{z}$ be the generalized eigenspace of $\bar{L}_{0}$ with eigenvalue $-z \in \mathbb{C}$. There exist $z_{1}, \cdots, z_{m} \in \mathbb{Q}$ such that

$$
\left\{z \mid V_{z} \neq 0\right\} \subset\left\{z_{1}-\mathbb{N}\right\} \cup \cdots \cup\left\{z_{m}-\mathbb{N}\right\} .
$$

Then $V=\bigoplus_{z \in \mathbb{C}} V_{z}$ with $\operatorname{dim} V_{z}<\infty$ becomes a $\mathbb{Q}$-graded $\hat{\mathfrak{g}}_{\bar{k}}$-representation, i.e. $x_{(n)} V_{z} \subset V_{z+n}$ for any $x_{(n)} \in \hat{\mathfrak{g}}$ (see [KL1, Lemma 2.20, Proposition 2.21]). In case $V=V_{\lambda, \bar{k}}$ is a Weyl module, $\bar{L}_{0}$ acts on $V_{\lambda, \bar{k}}$ semisimply. More specifically, we have

$$
\left.\bar{L}_{0}\right|_{U\left(\hat{\mathfrak{g}}_{<0}\right)_{-n} \otimes V_{\lambda}}=-\frac{\langle\lambda, \lambda+2 \rho\rangle}{2 \varkappa}+n,
$$


where $\rho$ is the half sum of positive roots.

The dual representation of $V$ is defined to be $V^{*}=\bigoplus_{z}\left(V_{z}\right)^{*}$, where the $\hat{\mathfrak{g}}$-action is given by $X f(v)=f(-X v)$ for any $X \in \hat{\mathfrak{g}}, f \in V^{*}, v \in V$. In particular, $V^{*}$ is a $\hat{\mathfrak{g}}_{-\bar{k}}$-module and locally $U\left(\hat{\mathfrak{g}}_{<0}\right)$-finite. To give $V^{*}$ the structure of a graded $\hat{\mathfrak{g}}$ module as well, set $\left(V^{*}\right)_{z}=\left(V_{-z}\right)^{*}$ or, equivalently, set $\left(V^{*}\right)_{z}$ to be the generalized $(-z)$-eigenspace of the operator

$$
L_{0}^{\prime}=\frac{1}{\varkappa} \sum_{j>0} \sum_{i} \tau_{i(j)} \tau_{i(-j)}+\frac{1}{2 \varkappa} \sum_{i} \tau_{i(0)} \tau_{i(0)}
$$

which acts on $V^{*}$.

The contragredient dual $V^{c}$ is isomorphic to $V^{*}$ as a vector space, but instead of using -Id $: \hat{\mathfrak{g}} \rightarrow \hat{\mathfrak{g}}$, we use the anti-involution $x_{(n)} \mapsto-x_{(-n)}, \underline{c} \mapsto \underline{c}$ to define the $\hat{\mathfrak{g}}$-action on $V^{c}$. Unlike $V^{*}$, the contragredient module $V^{c}$ is a $\hat{\mathfrak{g}}_{\bar{k}}$-representation and locally $U\left(\hat{\mathfrak{g}}_{>0}\right)$-finite. In fact, it belongs to $\mathcal{O}_{-\varkappa}$.

Given $V \in \mathcal{O}_{-\varkappa}$, define a map $\rho_{V}: V^{*} \otimes V \rightarrow U(\hat{\mathfrak{g}}, \bar{k})^{*}$ by

$$
\rho_{V}(f \otimes v)(u)=f(u \cdot v)
$$

for any $f \in V^{*}, v \in V, u \in U(\hat{\mathfrak{g}})$. It is easy to see that $\rho_{V}$ is a $\hat{\mathfrak{g}}_{-\bar{k}} \oplus \hat{\mathfrak{g}}_{\bar{k}}$-map. The $\hat{\mathfrak{g}}_{-\bar{k}} \oplus \hat{\mathfrak{g}}_{\bar{k}}$-module structure of $U(\hat{\mathfrak{g}}, \bar{k})^{*}$ is given by

$$
(X, 0) \cdot g=-g \cdot X, \quad(0, X) \cdot g=X \cdot g
$$

for any $X \in \hat{\mathfrak{g}}, g \in U(\hat{\mathfrak{g}}, \bar{k})^{*}$. We denote the image of $\rho_{V}$ by $\mathbb{M}(V)$, called the matrix coefficients of $V$.

Recall the $\hat{\mathfrak{g}}_{-\bar{k}} \oplus \hat{\mathfrak{g}}_{\bar{k}}$-map,

$$
\Phi: S_{\gamma} \otimes_{U} \mathbb{V} \rightarrow U(\hat{\mathfrak{g}}, \bar{k})^{*},
$$

defined earlier. As pointed out in Remark 3.8, the map $\Phi$ is injective and its image $\operatorname{Im} \Phi$ is $U\left(\hat{\mathfrak{g}}_{<0}\right)^{\circledast} \otimes U(\mathfrak{g})_{\text {Hopf }}^{*} \otimes U\left(\hat{\mathfrak{g}}_{>0}\right)^{\circledast}$. Here

$$
U\left(\hat{\mathfrak{g}}_{<0}\right)^{\circledast}=\bigoplus_{n \leq 0}\left(U\left(\hat{\mathfrak{g}}_{<0}\right)_{n}\right)^{*}, \quad U\left(\hat{\mathfrak{g}}_{>0}\right)^{\circledast}=\bigoplus_{n \geq 0}\left(U\left(\hat{\mathfrak{g}}_{>0}\right)_{n}\right)^{*}
$$

are graded duals.

Proposition 4.3. The space $\operatorname{Im} \Phi$ consists of matrix coefficients of modules from the category $\mathcal{O}_{-\varkappa}$, i.e. $\operatorname{Im} \Phi=\sum_{V \in \mathcal{O}_{-\varkappa}} \mathbb{M}(V)$.

Proof. Let $\left(V=\bigoplus_{z} V_{z}\right) \in \mathcal{O}_{-\varkappa}, v \in V$, and $f \in V^{*}$. For any $u=u_{<0} u_{0} u_{>0} \in U(\hat{\mathfrak{g}})$ with $u_{>0} \in U\left(\hat{\mathfrak{g}}_{>0}\right), u_{0} \in U(\mathfrak{g}), u_{<0} \in U\left(\hat{\mathfrak{g}}_{<0}\right)$, we have by definition

$$
\rho_{V}(f \otimes v)(u)=\left\langle f, u_{<0} u_{0} u_{>0} \cdot v\right\rangle=\left\langle\overline{u_{<0}} \cdot f, u_{0} \cdot u_{>0} \cdot v\right\rangle .
$$

Since $V \in \mathcal{O}_{-\varkappa}$, there exist $n_{1}, n_{2} \in \mathbb{N}$ such that $U\left(\hat{\mathfrak{g}}_{<0}\right)_{-n_{1}} \cdot f=U\left(\hat{\mathfrak{g}}_{>0}\right)_{n_{2}} \cdot v=0$. Moreover, each $V_{z}$ is finite-dimensional and semisimple as a $\mathfrak{g}$-module, therefore it follows that

$$
\phi_{V}(f \otimes v) \in U\left(\hat{\mathfrak{g}}_{<0}\right)^{\circledast} \otimes U(\mathfrak{g})_{\text {Hopf }}^{*} \otimes U\left(\hat{\mathfrak{g}}_{>0}\right)^{\circledast}
$$

i.e. $\mathbb{M}(V) \subset \operatorname{Im} \Phi$.

On the other hand, for any $g \in \operatorname{Im} \Phi$ there exists an $n \in \mathbb{N}$ such that $U\left(\hat{\mathfrak{g}}_{>0}, \bar{k}\right)_{n}$. $g=0$. The $\hat{\mathfrak{g}}_{\geq 0}$-submodule generated by $g$ is a nil-module. Hence, the $\hat{\mathfrak{g}}$-submodule $W=U(\hat{\mathfrak{g}}, \bar{k}) \cdot g$ generated by $g$ is a quotient of a generalized Weyl module, which implies that $W$ belongs to $\mathcal{O}_{-\varkappa}$. Let $\delta$ be the functional on $U^{*}$ defined by $\delta\left(g^{\prime}\right)=$ $g^{\prime}(1)$. Then $\delta \in W^{*}$ and $g=\rho_{W}(\delta \otimes g) \in \mathbb{M}(W)$. 
Define two operators $\bar{L}_{0}, L_{0}^{\prime}$ acting on $\operatorname{Im} \Phi$ as follows: For any $g \in \operatorname{Im} \Phi$, set

$$
\bar{L}_{0} g=-\frac{1}{\varkappa} \sum_{j>0} \sum_{i} \tau_{i(-j)} \cdot \tau_{i(j)} \cdot g-\frac{1}{2 \varkappa} \sum_{i} \tau_{i(0)} \cdot \tau_{i(0)} \cdot g
$$

and

$$
L_{0}^{\prime} g=\frac{1}{\varkappa} \sum_{j>0} \sum_{i} g \cdot \tau_{i(-j)} \cdot \tau_{i(j)}+\frac{1}{2 \varkappa} \sum_{i} g \cdot \tau_{i(0)} \cdot \tau_{i(0)} .
$$

Let $(\operatorname{Im} \Phi)_{z^{\prime}, z}$ be the subspace consisting of all $g \in \operatorname{Im} \Phi$ such that $g$ is in the kernel of some power of $\bar{L}_{0}+z \mathrm{Id}$ and the kernel of some power of $L_{0}^{\prime}+z^{\prime} \mathrm{Id}$. Then

$$
\operatorname{Im} \Phi=\bigoplus_{z, z^{\prime}}(\operatorname{Im} \Phi)_{z^{\prime}, z} \quad \text { and } \quad \rho_{V}\left(\left(V^{*}\right)_{z^{\prime}} \otimes V_{z}\right) \subset(\operatorname{Im} \Phi)_{z^{\prime}, z}
$$

for any $V \in \mathcal{O}_{-\varkappa}$. Moreover, one has

$$
(\operatorname{Im} \Phi)_{z^{\prime}, z} \cdot x_{(n)} \subset(\operatorname{Im} \Phi)_{z^{\prime}+n, z} \quad \text { and } \quad x_{(n)} \cdot(\operatorname{Im} \Phi)_{z^{\prime}, z} \subset(\operatorname{Im} \Phi)_{z^{\prime}, z+n}
$$

for any $x_{(n)} \in \hat{\mathfrak{g}}$. Define a $\mathbb{Z}$-grading on $\operatorname{Im} \Phi$ as follows: For any $g_{1} \in\left(U\left(\hat{\mathfrak{g}}_{<0}\right)_{n_{1}}\right)^{*}$, $a \in U(\mathfrak{g})_{\text {Hopf }}^{*}$, and $g_{2} \in\left(U\left(\hat{\mathfrak{g}}_{>0}\right)_{n_{2}}\right)^{*}$, set

$$
\operatorname{deg}\left(g_{1} \otimes a \otimes g_{2}\right)=-n_{1}-n_{2} .
$$

Denote $(\operatorname{Im} \Phi)_{n}=\{g \mid \operatorname{deg} g=n\}$. It is not difficult to see that

$$
(\operatorname{Im} \Phi)_{n}=\bigoplus_{z+z^{\prime}=n}(\operatorname{Im} \Phi)_{z^{\prime}, z} .
$$

Following [KL1, 3.3], for any $\lambda, \mu \in P^{+}$we say that $\lambda \leq \mu$ if either $\lambda=\mu$ or

$$
\langle\lambda, \lambda+2 \rho\rangle<\langle\mu, \mu+2 \rho\rangle .
$$

Let $\mathcal{O}_{-\varkappa}^{s}$ be the full subcategory of $\mathcal{O}_{-\varkappa}$ whose objects are the $V$ in $\mathcal{O}_{-\varkappa}$ such that the composition factors of $V$ are of the form $L_{\lambda, \bar{k}}$ for some $\lambda$ in the finite set

$$
F^{s}=\left\{\lambda \in P^{+} \mid\langle\lambda, \lambda+2 \rho\rangle \leq s\right\} .
$$

We say that a module $V \in \mathcal{O}_{-\varkappa}$ is tilting if both $V$ and $V^{c}$ admit a Weyl flag. For any $\lambda \in P^{+}$, there exists an indecomposable tilting module $T_{\lambda, \bar{k}}$ such that the Weyl filtration starts with $V_{\lambda, \bar{k}} \hookrightarrow T_{\lambda, \bar{k}}$, and any other Weyl modules $V_{\mu, \bar{k}}$ entering the Weyl filtration of $T_{\lambda, \bar{k}}$ satisfy $\mu<\lambda$ ([KL4, Proposition 27.2]).

Lemma 4.4. Let $V, V^{\prime} \in \mathcal{O}_{-\varkappa}$.

(1) If $V$ admits a (finite) Weyl filtration with factors isomorphic to $V_{\lambda_{i}, \bar{k}}$ for some $\lambda_{i} \in P^{+}$, then $\mathbb{M}(V) \subset \sum_{i} \mathbb{M}\left(T_{\lambda_{i}, \bar{k}}\right)$.

(2) If $V^{\prime}$ admits a (finite) filtration with factors isomorphic to $V_{\mu_{i}, \bar{k}}^{c}$ for some $\mu_{i} \in P^{+}$, then $\mathbb{M}\left(V^{\prime}\right) \subset \sum_{i} \mathbb{M}\left(T_{\mu_{i}, \bar{k}}^{c}\right)$.

Proof. The proof is exactly the same as that of [Z2, Lemma 3.2]. We can construct an injection $V \hookrightarrow \bigoplus_{i} T_{\lambda_{i}, \bar{k}}$ or a surjection $\bigoplus_{i} T_{\mu_{i}, \bar{k}}^{c} \rightarrow V^{\prime}$, since $\operatorname{Ext}_{\mathcal{O}_{-\varkappa}}^{1}\left(V_{\lambda, \bar{k}}, V_{\mu, \bar{k}}^{c}\right)$ $=0($ [KL4 , Proposition 27.1]).

Corollary 4.5. The space $\operatorname{Im} \Phi$ consists of the matrix coefficients of tilting modules from $\mathcal{O}_{-\varkappa}$, i.e. $\operatorname{Im} \Phi=\sum_{T \in \mathcal{O}_{-\varkappa}, T \text { tilting }} \mathbb{M}(T)$. 
Proof. For any $V \in \mathcal{O}_{-\varkappa}$, choose $s$ such that $V \in \mathcal{O}_{-\varkappa}^{s}$. By [KL1, Proposition 3.9], there exists a $P$, having a finite Weyl flag and projective in $\mathcal{O}_{-\varkappa}^{s}$, such that $V$ a quotient of $P$. Hence by Lemma 4.4 (1), we have $\mathbb{M}(V) \subset \mathbb{M}(P) \subset \sum_{i} \mathbb{M}\left(T_{\lambda_{i}, \bar{k}}\right)$ for some $\lambda_{i} \in F^{s}$.

Proposition 4.6. Order the dominant weights $P^{+}=\left\{\nu_{1}, \cdots, \nu_{i}, \cdots\right\}$ in such a way that $\nu_{i}<\nu_{j}$ implies $i<j$. Set $\Lambda^{i}=\sum_{j \leq i} \mathbb{M}\left(T_{\nu_{j}, \bar{k}}\right)$. Then $\Lambda^{1} \subset \cdots \subset$ $\Lambda^{i-1} \subset \Lambda^{i} \subset \cdots$ is an increasing filtration of $\hat{\mathfrak{g}}_{-\bar{k}} \oplus \hat{\mathfrak{g}}_{\bar{k}}$-submodules of $\operatorname{Im} \Phi$ with factors $\Lambda^{i} / \Lambda^{i-1}$ isomorphic to $V_{\nu_{i}, \bar{k}}^{*} \otimes V_{-\omega_{0} \nu_{i}, \bar{k}}^{c}$, where $\omega_{0}$ is the longest element in the Weyl group.

Proof. The proof is the same as that of [Z2, Theorem 3.3], using Lemma 4.4.

Remark 4.7. The category $\mathcal{O}_{-\varkappa}$ is a direct sum of subcategories corresponding to the orbits of the shifted action of affine Weyl group on the weight lattice ([KL4, Lemma 27.7]). We can decompose $\operatorname{Im} \Phi$, as a $\hat{\mathfrak{g}}_{-\bar{k}} \oplus \hat{\mathfrak{g}}_{\bar{k}}$-module, into summands corresponding to the orbits as well. Then each summand has an increasing filtration of the above type.

Proposition 4.8. The vertex operator algebra $\mathbb{V}$ is isomorphic to $\mathcal{H}$ om ${ }_{U}\left(S_{\gamma}, \operatorname{Im} \Phi\right)$ as a $\hat{\mathfrak{g}}_{k} \oplus \hat{\mathfrak{g}}_{\bar{k}}$-module.

Proof. Recall that $\operatorname{Im} \Phi \cong S_{\gamma} \otimes_{U} \mathbb{V}=N^{\circledast} \otimes \mathbb{B}$. Hence we have

$$
\mathcal{H} \operatorname{om}_{U}\left(S_{\gamma}, \operatorname{Im} \Phi\right) \cong \mathcal{H}^{\circ} m_{N}\left(N^{\circledast}, N^{\circledast} \otimes \mathbb{B}\right) \cong \mathcal{H}_{\mathbb{C}}\left(N^{\circledast}, \mathbb{B}\right) \cong N \otimes \mathbb{B} \cong \mathbb{V}
$$

because $\mathbb{B}$ is non-positively graded while $N^{\circledast}$ is non-negatively graded. Moreover, the induced isomorphism

$$
\mathbb{V} \rightarrow \mathcal{H o m}_{U}\left(S_{\gamma}, \operatorname{Im} \Phi\right) \cong \mathcal{H}_{U}\left(S_{\gamma}, S_{\gamma} \otimes_{U} \mathbb{V}\right)
$$

is a $\hat{\mathfrak{g}} \oplus \hat{\mathfrak{g}}$-map.

Lemma 4.9. For any $b \in \mathbb{B}$, there exists an $i$ such that $\Phi\left(N^{\circledast} \otimes b\right) \subset \Lambda^{i}$.

Proof. For any $f \in N^{\circledast}$ and $u_{\geq 0} \in U\left(\hat{\mathfrak{g}}_{\geq 0}\right)$, we have $u_{\geq 0}^{2} \cdot(f \otimes b)=f \otimes\left(u_{\geq 0}^{2} \cdot b\right)$. Let $\mathcal{N}$ be the $U\left(\hat{\mathfrak{g}}_{\geq 0}, \bar{k}\right)$-submodule of $\mathbb{B}$ generated by $b$. Then $\mathcal{N}$ is a nil-module and the $\hat{\mathfrak{g}}_{\bar{k}}$-submodule $U(\hat{\mathfrak{g}}, \bar{k}) \cdot(f \otimes b)$ generated by $f \otimes b$ is a quotient of the generalized Weyl module $\mathcal{N}_{\bar{k}}$. Hence $\Phi(f \otimes b) \in \mathbb{M}\left(\mathcal{N}_{\bar{k}}\right)$ for any $f \in N^{\circledast}$, and we conclude that there exists an $i$ such that $\Phi\left(N^{\circledast} \otimes b\right) \subset \Lambda^{i}$.

Theorem 4.10. Set $\Sigma^{i}=\mathcal{H} \operatorname{om}_{U}\left(S_{\gamma}, \Lambda^{i}\right)$. Then $\mathbb{V}=\bigcup_{i} \Sigma^{i}$ and $\Sigma^{1} \subset \cdots \subset \Sigma^{i-1} \subset$ $\Sigma^{i} \subset \cdots$ is an increasing filtration of $\hat{\mathfrak{g}}_{k} \oplus \hat{\mathfrak{g}}_{\bar{k}}$-submodules of $\mathbb{V}$ with factors $\Sigma^{i} / \Sigma^{i-1}$ isomorphic to $V_{-\omega_{0} \nu_{i}, k} \otimes V_{-\omega_{0} \nu_{i}, \bar{k}}^{c}$.

Proof. For any $u_{<0} \otimes b \in N \otimes \mathbb{B} \cong \mathbb{V}$, let $\mathcal{N}^{\prime} \subset \mathbb{B}$ be the $U\left(\hat{\mathfrak{g}}_{\geq 0}, k\right)$-submodule generated by $b$. Then $\mathcal{N}^{\prime}$ is finite-dimensional. For any $s \in S_{\gamma}$ we have

$$
p\left(s \otimes\left(u_{<0} \otimes b\right)\right) \in N^{\circledast} \otimes \mathcal{N}^{\prime},
$$

where $p: S_{\gamma} \otimes \mathbb{V} \rightarrow S_{\gamma} \otimes_{U} \mathbb{V}$ is the canonical projection. By Lemma 4.9, there exists an $i$ such that $p\left(s \otimes\left(u_{<0} \otimes b\right)\right) \in \Lambda^{i}$ for any $s \in S_{\gamma}$, hence

$$
u_{<0} \otimes b \in \mathcal{H o m}_{U}\left(S_{\gamma}, \Lambda^{i}\right)=\Sigma^{i} .
$$

This proves that $\mathbb{V}=\bigcup_{i} \Sigma^{i}$. 
Note that $\Lambda^{i}=\bigoplus_{z^{\prime}, z} \Lambda_{z^{\prime}, z}^{i}$ with $\operatorname{dim} \Lambda_{z^{\prime}, z}^{i}<\infty$. Fix $z$, the exact sequence of $\hat{\mathfrak{g}}_{-\bar{k}} \oplus \hat{\mathfrak{g}}_{\bar{k}}$-modules

$$
0 \rightarrow \Lambda^{i-1} \rightarrow \Lambda^{i} \rightarrow V_{\nu_{i}, \bar{k}}^{*} \otimes V_{-\omega_{0} \nu_{i}, \bar{k}}^{c} \rightarrow 0
$$

restricts to an exact sequence of $\hat{\mathfrak{g}}_{-\bar{k}}$-modules

$$
0 \rightarrow \bigoplus_{z^{\prime}} \Lambda_{z^{\prime}, z}^{i-1} \rightarrow \bigoplus_{z^{\prime}} \Lambda_{z^{\prime}, z}^{i} \rightarrow V_{\nu_{i}, \bar{k}}^{*} \otimes\left(V_{-\omega_{0} \nu_{i}, \bar{k}}^{c}\right)_{z} \rightarrow 0 .
$$

Since $V_{\nu_{i}, \bar{k}}^{*} \otimes\left(V_{-\omega_{0} \nu_{i}, \bar{k}}^{c}\right)_{z}$ is isomorphic to a finite direct sum of grading-shifted copies of $N^{\circledast}$ over $N$, by induction on $i$, so is $\bigoplus_{z^{\prime}} \Lambda_{z^{\prime}, z}^{i}$ for each $i$. The two exact sequences split over $N$, which means that there exists a grading preserving $N$-map

$$
V_{\nu_{i}, \bar{k}}^{*} \otimes V_{-\omega_{0} \nu_{i}, \bar{k}}^{c} \rightarrow \Lambda^{i}
$$

such that its composition with the projection is the identity on the former. Since

$$
\mathcal{H o m}_{U}\left(S_{\gamma},-\right) \cong \mathcal{H o m}_{N}\left(N^{\circledast},-\right),
$$

the sequence of $\hat{\mathfrak{g}}_{k} \oplus \hat{\mathfrak{g}}_{\bar{k}}$-modules

$$
0 \rightarrow \mathcal{H}_{U}\left(S_{\gamma}, \Lambda^{i-1}\right) \rightarrow \mathcal{H} \operatorname{om}_{U}\left(S_{\gamma}, \Lambda^{i}\right) \rightarrow \mathcal{H} \operatorname{om}_{U}\left(S_{\gamma}, V_{\nu_{i}, \bar{k}}^{*} \otimes V_{-\omega_{0} \nu_{i}, \bar{k}}^{c}\right) \rightarrow 0
$$

is exact. Hence we have

$$
\Sigma^{i} / \Sigma^{i-1} \cong \mathcal{H} \operatorname{om}_{U}\left(S_{\gamma}, V_{\nu_{i}, \bar{k}}^{*} \otimes V_{-\omega_{0} \nu_{i}, \bar{k}}^{c}\right),
$$

which is isomorphic to $V_{-\omega_{0} \nu_{i}, k} \otimes V_{-\omega_{0} \nu_{i}, \bar{k}}^{c}$ by Proposition 2.9, Lemma2.10, and the fact that the grading on $V_{-\omega_{0} \nu_{i}, \bar{k}}^{c}$ is bounded from above.

Remark 4.11. The decomposition of $\operatorname{Im} \Phi$ discussed in Remark 4.7 also leads to a decomposition of $\mathbb{V}$ into summands corresponding to the orbits of the affine Weyl group. Each summand has an increasing filtration of the above type.

Corollary 4.12. The vertex operator algebra $\mathbb{V}$ admits a decreasing filtration of $\hat{\mathfrak{g}}_{k} \oplus \hat{\mathfrak{g}}_{\bar{k}}$-submodules $\mathbb{V} \supset \Xi_{1} \supset \cdots \supset \Xi_{i-1} \supset \Xi_{i} \supset \cdots$ with factors $\Xi_{i-1} / \Xi_{i}$ isomorphic to $V_{-\omega_{0} \nu_{i}, k}^{c} \otimes V_{-\omega_{0} \nu_{i}, \bar{k}}$ and $\bigcap_{i} \Xi_{i}=0$.

Proof. Let $L_{0}, \bar{L}_{0}: \mathbb{V} \rightarrow \mathbb{V}$ be the Sugawara operators associated to the $\hat{\mathfrak{g}}_{k^{-}}$and $\hat{\mathfrak{g}}_{\bar{k}}$-actions on $\mathbb{V}$, respectively, i.e.

$$
L_{0}=\frac{1}{\varkappa} \sum_{j>0} \sum_{i} \tau_{i(-j)} \tau_{i(j)}+\frac{1}{2 \varkappa} \sum_{i} \tau_{i(0)} \tau_{i(0)}
$$

and

$$
\bar{L}_{0}=-\frac{1}{\varkappa} \sum_{j>0} \sum_{i} \bar{\tau}_{i(-j)} \bar{\tau}_{i(j)}-\frac{1}{2 \varkappa} \sum_{i} \bar{\tau}_{i(0)} \bar{\tau}_{i(0)} .
$$

If we regard the vertex operator algebra $\mathbb{V}=\bigoplus_{n \geq 0} \mathbb{V}_{n}$ as non-negatively graded, then the sum $\mathcal{L}_{0}=L_{0}+\bar{L}_{0}$ acts as the gradation operator i.e. $\left.\mathcal{L}_{0}\right|_{\mathbb{V}_{n}}=n$ Id (see [Z1, Proposition 3.18, 3.22]). Let $\mathbb{V}_{z_{1}, z_{2}}$ be the subspace consisting of $v \in \mathbb{V}$ such that $v$ is killed by some power of $L_{0}-z_{1}$ Id and some power of $\bar{L}_{0}-z_{2}$ Id. It follows from Theorem 4.10 that $\mathbb{V}=\bigoplus_{z_{1}, z_{2}} \mathbb{V}_{z_{1}, z_{2}}$ and $\operatorname{dim} \mathbb{V}_{z_{1}, z_{2}}<\infty$.

Recall the symmetric non-degenerate bilinear form $\langle\rangle:, \mathbb{V} \times \mathbb{V} \rightarrow \mathbb{C}$ constructed in [Z1, 3.26]. It was shown to be compatible with the VOA structure of $\mathbb{V}$, so we 
have $x_{(n)}^{*}=-x_{(-n)}$ and $\bar{y}_{(n)}^{*}=-\bar{y}_{(-n)}$ for any $x_{(n)} \in \hat{\mathfrak{g}}_{k}$ and $\bar{y}_{(n)} \in \hat{\mathfrak{g}}_{\bar{k}}$. This implies that $L_{0}^{*}=L_{0}$ and $\bar{L}_{0}^{*}=\bar{L}_{0}$, i.e.

$$
\left\langle L_{0} \cdot, \cdot\right\rangle=\left\langle\cdot, L_{0} \cdot\right\rangle \quad \text { and } \quad\left\langle\bar{L}_{0} \cdot, \cdot\right\rangle=\left\langle\cdot, \bar{L}_{0} \cdot\right\rangle .
$$

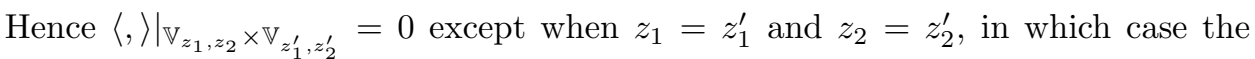
pairing is non-degenerate. Let $\mathbb{V}^{c}=\bigoplus_{z_{1}, z_{2}} \mathbb{V}_{z_{1}, z_{2}}^{*}$ be the contragredient dual of $\mathbb{V}$, where both the $\hat{\mathfrak{g}}_{k^{-}}$and $\hat{\mathfrak{g}}_{\bar{k}^{-}}$-actions on $\mathbb{V}^{c}$ are defined by the anti-involution $x_{(n)} \mapsto-x_{(-n)} ; \underline{c} \mapsto \underline{c}$ of $\hat{\mathfrak{g}}$. Then we have an isomorphism of $\hat{\mathfrak{g}}_{k} \oplus \hat{\mathfrak{g}}_{\bar{k}}$-modules $\mathbb{V} \cong \mathbb{V}^{c}$ induced by the bilinear form $\langle$,$\rangle .$

Define

$$
\Xi_{i}=\left\{v \in \mathbb{V} \mid\left\langle v, \Sigma_{i}\right\rangle=0\right\} .
$$

Then $\Xi_{i}$ is a $\hat{\mathfrak{g}}_{k} \oplus \hat{\mathfrak{g}}_{\bar{k}}$-submodule of $\mathbb{V}$ and $\Xi_{i} \subset \Xi_{i-1}$. Since $\bigcup_{i} \Sigma_{i}=\mathbb{V}$ and $\langle$,$\rangle is$ non-degenerate, we have $\bigcap_{i} \Xi_{i}=0$. Moreover, we have the following isomorphisms of $\hat{\mathfrak{g}}_{k} \oplus \hat{\mathfrak{g}}_{\bar{k}}$-modules:

$$
\Xi_{i-1} / \Xi_{i} \cong\left(\Sigma_{i} / \Sigma_{i-1}\right)^{c} \cong\left(V_{-\omega_{0} \nu_{i}, k} \otimes V_{-\omega_{0} \nu_{i}, \bar{k}}^{c}\right)^{c} \cong V_{-\omega_{0} \nu_{i}, k}^{c} \otimes V_{-\omega_{0} \nu_{i}, \bar{k}} .
$$

The total central charge $-2 h^{\vee}$ of $\mathbb{V}$ with respect to the diagonal action of $\hat{\mathfrak{g}}$ allows us to introduce the semi-infinite cohomology of $\hat{\mathfrak{g}}$ with coefficients in $\mathbb{V}$, denoted by

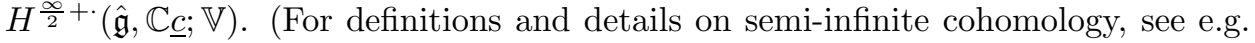
F, FGZ.)

Recall that $A=\mathbb{V}_{0}$ is the space of regular functions on $G$; we consider the diago-

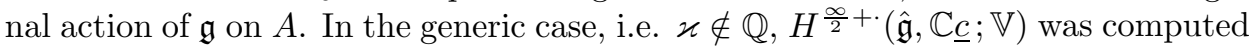
in [FS]; it is isomorphic to the Lie algebra cohomology of $\mathfrak{g}$ with coefficients in $A$. Here, we extend this result to the case of non-critial rational levels.

Corollary 4.13. The semi-infinite cohomology of $\hat{\mathfrak{g}}$ with coefficients in $\mathbb{V}$ is isomorphic to the Lie algebra cohomology of $\mathfrak{g}$ with coefficients in the regular functions of $G$, i.e.

$$
H^{\frac{\infty}{2}+\cdot}(\hat{\mathfrak{g}}, \mathbb{C} \underline{\underline{C}} ; \mathbb{V})=H^{\cdot}(\mathfrak{g}, A)=\mathbb{C}[P]^{W} \otimes H_{D R}^{\cdot}(G) .
$$

Here, $P$ is the weight lattice and $W$ is the Weyl group of $\mathfrak{g} ; \mathbb{C}[P]^{W}$ is the space of formal characters of finite-dimensional $\mathfrak{g}$-modules; $H_{D R}(G)$ is the holomorphic de Rham cohomology of the Lie group $G$.

Proof. The proof is the same as that of [FS, Theorem 2.9], where generic values of $\varkappa$ are considered. When $\varkappa$ is irrational, the $\hat{\mathfrak{g}} \oplus \overline{\hat{\mathfrak{g}}}$-module $\mathbb{V}$ is isomorphic to $\bigoplus_{\lambda \in P^{+}} V_{\lambda, k} \otimes V_{\lambda^{*}, \bar{k}}$; in particular it has a decomposition:

$$
\mathbb{V}=U\left(\hat{\mathfrak{g}}_{<0}\right) \otimes A \otimes U\left(\overline{\hat{\mathfrak{g}}}_{<0}\right) .
$$

The factor $U\left(\overline{\hat{\mathfrak{g}}}_{<0}\right)$ is identified with $U\left(\overline{\hat{\mathfrak{g}}}_{>0}\right)^{\circledast}$ via the non-degenerate (since $\varkappa$ is generic) contravariant pairing. Then one builds a filtration of the complex; the corresponding spectral sequence collapses, resulting in the semi-infinite cohomology groups being concentrated in the subspace of weight 0 .

In the case where $\varkappa$ is possibly rational (but nonzero), the module $\mathbb{V}$ does not have the decomposition as in the generic case. However, as we have shown in Theorem 4.10, $\mathbb{V}$ admits an increasing filtration of $\hat{\mathfrak{g}} \oplus \overline{\hat{\mathfrak{g}}}$-submodules with factors $V_{\lambda, k} \otimes V_{\lambda, \bar{k}}^{c}$ for $\lambda \in P^{+}$. Note that

$$
V_{\lambda, k} \otimes V_{\lambda, \bar{k}}^{c}=U\left(\hat{\mathfrak{g}}_{<0}\right) \otimes\left(V_{\lambda} \otimes V_{\lambda}^{*}\right) \otimes U\left(\overline{\mathfrak{g}}_{>0}\right)^{\circledast},
$$


where the factor $U\left(\overline{\hat{\mathfrak{g}}}_{>0}\right)^{\circledast}$ comes naturally from the contragredient module $V_{\lambda, \bar{k}}^{c}$. To be more specific, we identify $V_{\lambda, \bar{k}}^{c}$ with $V_{\lambda}^{*} \otimes U\left(\overline{\hat{\mathfrak{g}}}_{>0}\right)^{\circledast}$ by setting

$$
\left(v^{*} \otimes g\right)(P \otimes w)=v^{*}(w) g(\sigma P),
$$

where $v^{*} \in V_{\lambda}^{*}, g \in U\left(\overline{\hat{\mathfrak{g}}}_{>0}\right)^{\circledast}, w \in V_{\lambda}, P \in U\left(\overline{\hat{\mathfrak{g}}}_{<0}\right)$, and $\sigma$ is the anti-involution of $\hat{\mathfrak{g}}$ given by $x_{(n)} \mapsto-x_{(-n)} ; \underline{c} \mapsto \underline{c}$. Now, $\hat{\mathfrak{g}}_{<0}$ and $\overline{\hat{\mathfrak{g}}}_{>0}$ act in their respective factors $U\left(\hat{\mathfrak{g}}_{<0}\right)$ and $U\left(\overline{\mathfrak{\mathfrak { g }}}_{>0}\right)^{\circledast}$. The proof of Theorem 2.9 in [FS] applies and implies that

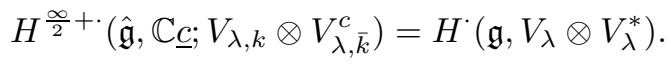

Thus, in the spectral sequence associated to the filtration of Theorem 4.10, nontrivial cohomology groups only appear in weight 0 . Hence, the semi-infinite cohomology of $\hat{\mathfrak{g}}$ with coefficients in $\mathbb{V}$ is reduced to the cohomology of $\mathfrak{g}$ with coefficients in $A$. The second equality $H^{\cdot}(\mathfrak{g}, A)=\mathbb{C}[P]^{W} \otimes H_{\mathrm{DR}}(G)$ is from classical Lie algebra cohomology theory.

\section{REFERENCES}

[A1] S. Arkhipov, Semi-infinite cohomology of quantum groups, Comm. Math. Phys. 188 (1997), no. 2, 379-405. MR1471820 (99e:16012)

[A2] S.M.Arkhipov, Semi-infinite cohomology of associative algebras and bar duality, Int. Math. Res. Not. 1997, no. 17, 833-863. MR1474841 (98j:16006)

[AG] S. Arkhipov, D. Gaitsgory, Differential operators on the loop group via chiral algebras, Int. Math. Res. Not. 2002, no. 4, 165-210. MR1876958 (2003f:20077)

[F] B. Feigin, Semi-infinite homology of Lie, Kac-Moody and Virasoro algebras, (Russian) Uspekhi Mat. Nauk 39 (1984), no. 2 (236), 195-196; English transl. in Russ. Math. Surv. 39, no. 2 (1984). MR740035 (85g:17003)

[FGZ] I. Frenkel, H. Garland, G. Zuckerman, Semi-infinite cohomology and string theory, Proc. Natl. Acad. Sci. USA 83 (1986), no. 22, 8442-8446. MR865483 (88d:17016)

[FS] I. Frenkel, K. Styrkas, Modified regular representations of affine and Virasoro algebras, VOA structure and semi-infinite cohomology, Adv. Math. 206 (2006), 57-111. MR.2261751 (2008d:17038)

[GMS1] V. Gorbounov, F. Malikov, V. Schechtman, Gerbes of chiral differential operators, II, Vertex algebroids, Invent. Math. 155 (2004), no. 3, 605-680. MR2038198 (2005e:17047)

[GMS2] V. Gorbounov, F. Malikov, V. Schechtman, On chiral differential operators over homogeneous spaces, Int. J. Math. Math. Sci. 26 (2001), no. 2, 83-106. MR.1836785|(2002g:14020)

[KL1] D. Kazhdan, G. Lusztig, Tensor structures arising from affine Lie algebras I, J. Amer. Math. Soc. 6 (1993), 905-947. MR1186962 (93m:17014)

[KL2] D. Kazhdan, G. Lusztig, Tensor structures arising from affine Lie algebras II, J. Amer. Math. Soc. 6 (1993), 949-1011. MR1186962 (93m:17014)

[KL3] D. Kazhdan, G. Lusztig, Tensor structures arising from affine Lie algebras III, J. Amer. Math. Soc. 7 (1994), 335-381. MR.1239506 (94g:17048)

[KL4] D. Kazhdan, G. Lusztig, Tensor structures arising from affine Lie algebras IV, J. Amer. Math. Soc. 7 (1994), 383-453. MR.1239507 (94g:17049)

[S] W. Soergel, Character formulas for tilting modules over Kac-Moody algebras, Represent. Theory, 2 (1998), 432-448. MR.1663141 (2000c:17048)

[V] A. Voronov, Semi-infinite homological algebra, Invent. Math. 113 (1993), 103-146. MR:1223226 (94f:17021)

[Z1] M. Zhu, Vertex operator algebras associated to modified regular representations of affine Lie algebras, Adv. Math. 219 (2008), 1513-1547. MR2458145(2009j:17026)

[Z2] M. Zhu, Regular representations of quantum groups at roots of unity, Int. Math. Res. Not., 2010, no. 15, 3039-3065. MR2673718

Department of Mathematics, 110 Frelinghuysen Rd, Rutgers University, Piscataway, NEW JERSEY 08854

E-mail address: minxian@math.rutgers.edu 